\title{
Direct Observation of Redox Induced Bubble Generation and Nanopore Formation Dynamics in Controlled Dielectric Breakdown
}

Ming Dong ${ }^{1}$, Zifan Tang ${ }^{1}$, Xiaodong $\mathrm{He}^{1}$, and Weihua Guan ${ }^{1,2^{*}}$

${ }^{1}$ Department of Electrical Engineering, Pennsylvania State University, University Park, Pennsylvania 16802, United States

${ }^{2}$ Department of Biomedical Engineering, Pennsylvania State University, University Park, Pennsylvania 16802, United States

* Corresponding Author, Email: w.guan@psu.edu, Tel: 1-814-867-5748

This PDF file includes:

- Figures S1 to S3

- Supporting Video S1 


\section{Supporting Figures}

(a)
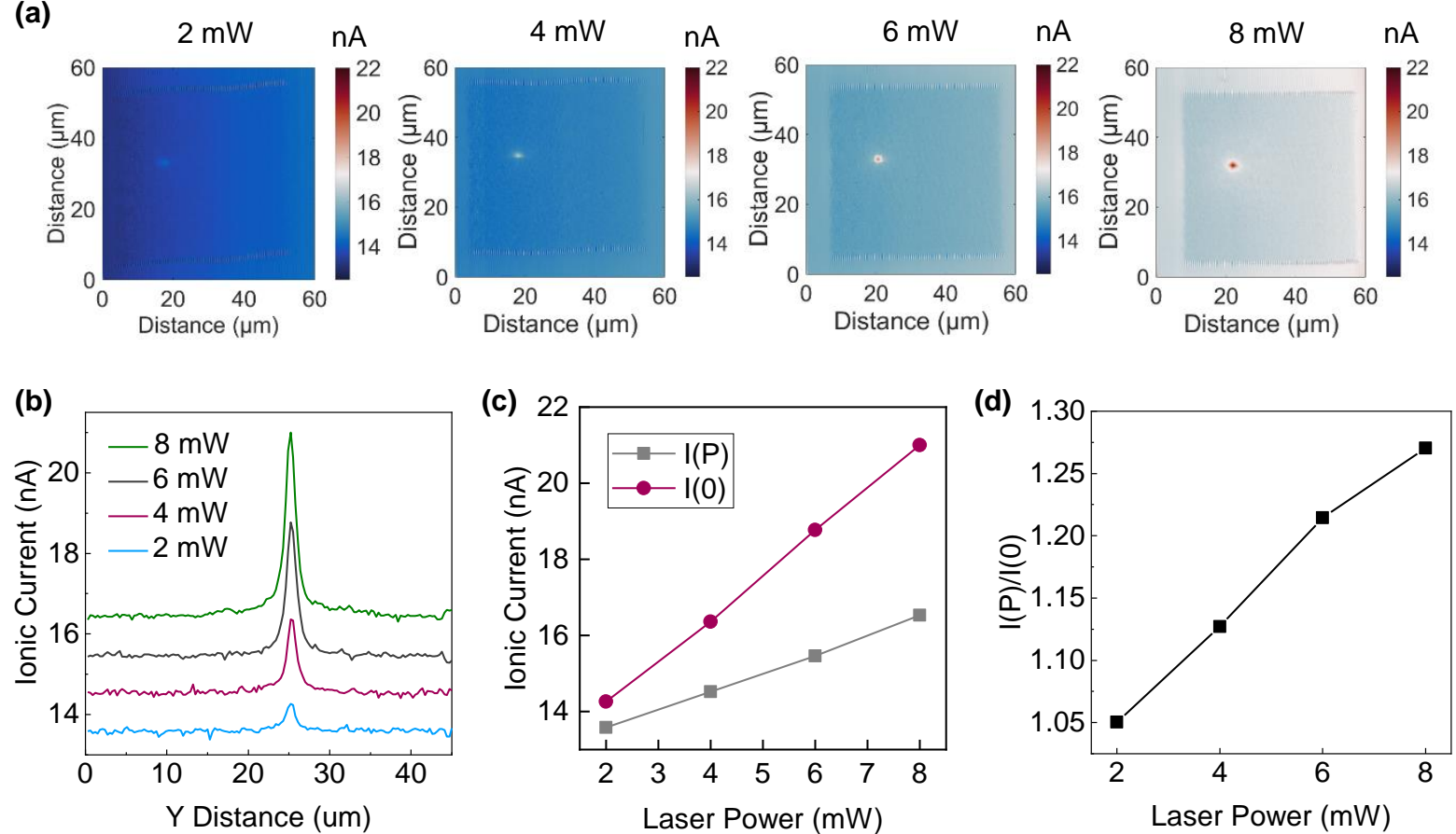

Figure S1. SNR relationship with different mapping laser power. (a) The laser enhanced ionic current mapping on TEM-drilled single nanopore at four different laser power ranging from 2 $\mathrm{mW}$ to $8 \mathrm{~mW}$. The enhanced area at nanopore is obvious under each laser power mapping. (b) Ionic current line profiles vertically through the nanopore at different laser power. (c) Peak intensity $\mathrm{I}(\mathrm{P})$, baseline $\mathrm{I}(0)$ at different laser power. The baseline of ionic current is shifting up with increasing laser power, which may result from the increasing solution temperature during mapping. The continuous heat accumulation from the joule heating effect and laser irradiation could increase the solution's temperature. It means the conductance of the solution is increasing during mapping, and the baseline is shifting up correspondingly. (d) The relationship between the signal to noise ratios (SNRs, I(P)/I(0)) and laser power. The SNRs for $2 \mathrm{~mW}, 4 \mathrm{~mW}, 6 \mathrm{~mW}$ and $8 \mathrm{~mW}$ laser are $1.04,1.13,1.21$ and 1.27 respectively. 
Supporting Information

(1) Pristine check

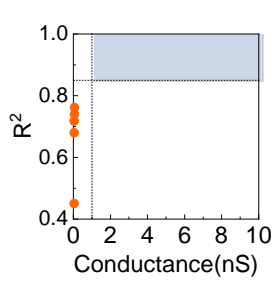

(2) Breakdown trial

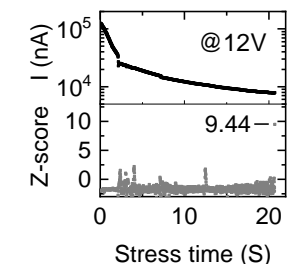

(3) Cross-verify

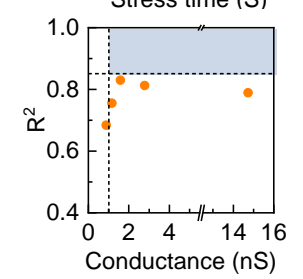

(4) Breakdown trial

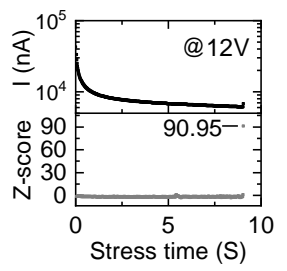

(5) Cross-verify

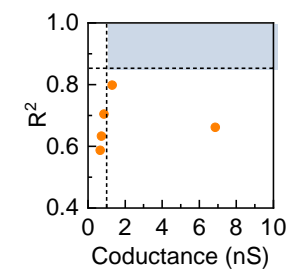

(6) Breakdown trial

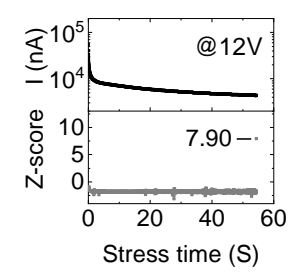

(7) Cross-verify
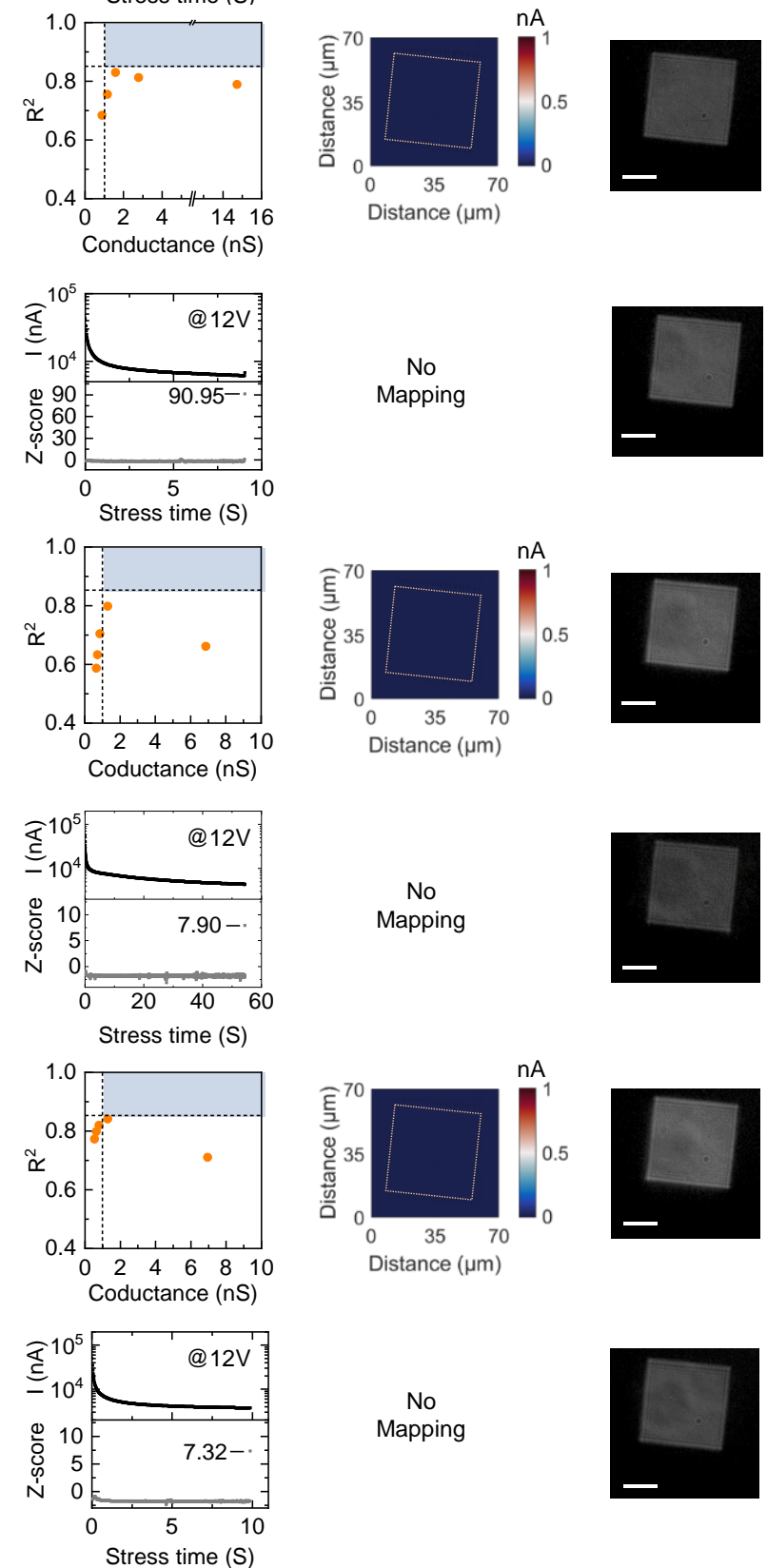

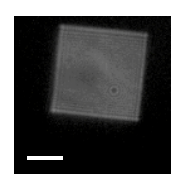

No Mapping

$$
\begin{gathered}
\text { No } \\
\text { Mapping }
\end{gathered}
$$
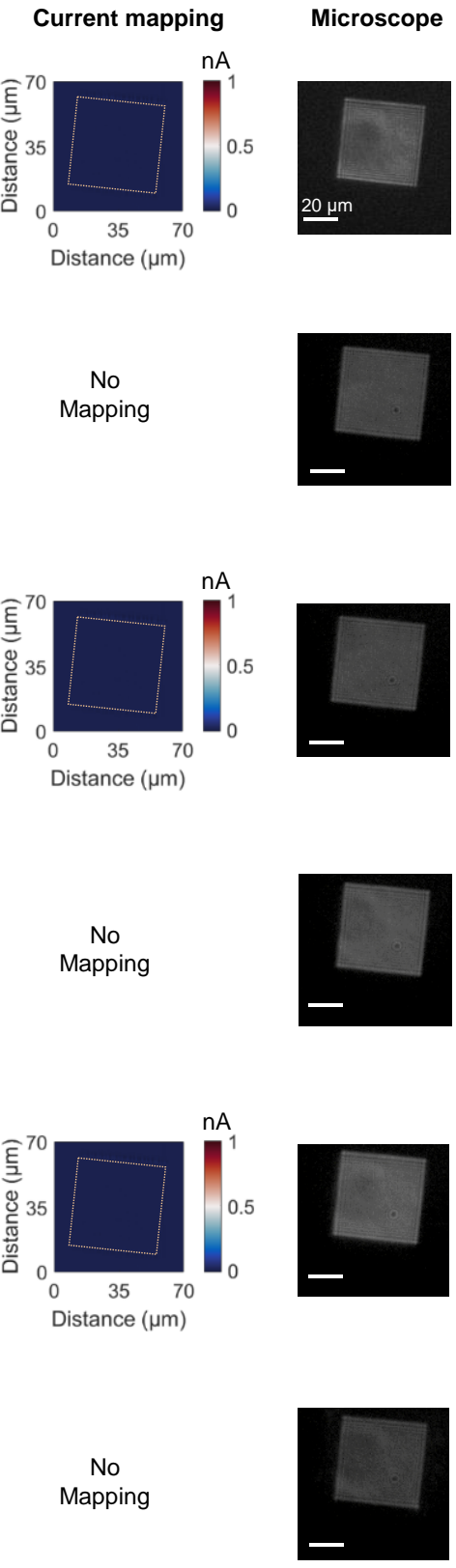

No Mapping

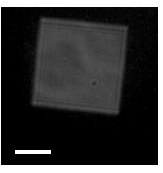


(9) Cross-verify

(10) Breakdown trial

(11) Cross-verify

(12) Breakdown trial

(13) Cross-verify

(14) Breakdown trial

(15) Cross-verify
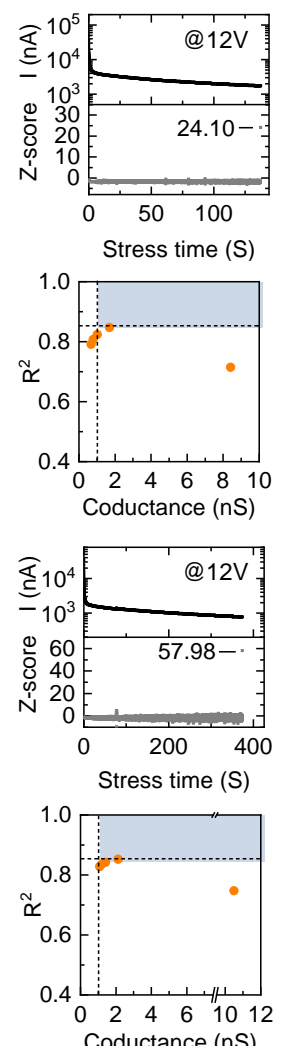
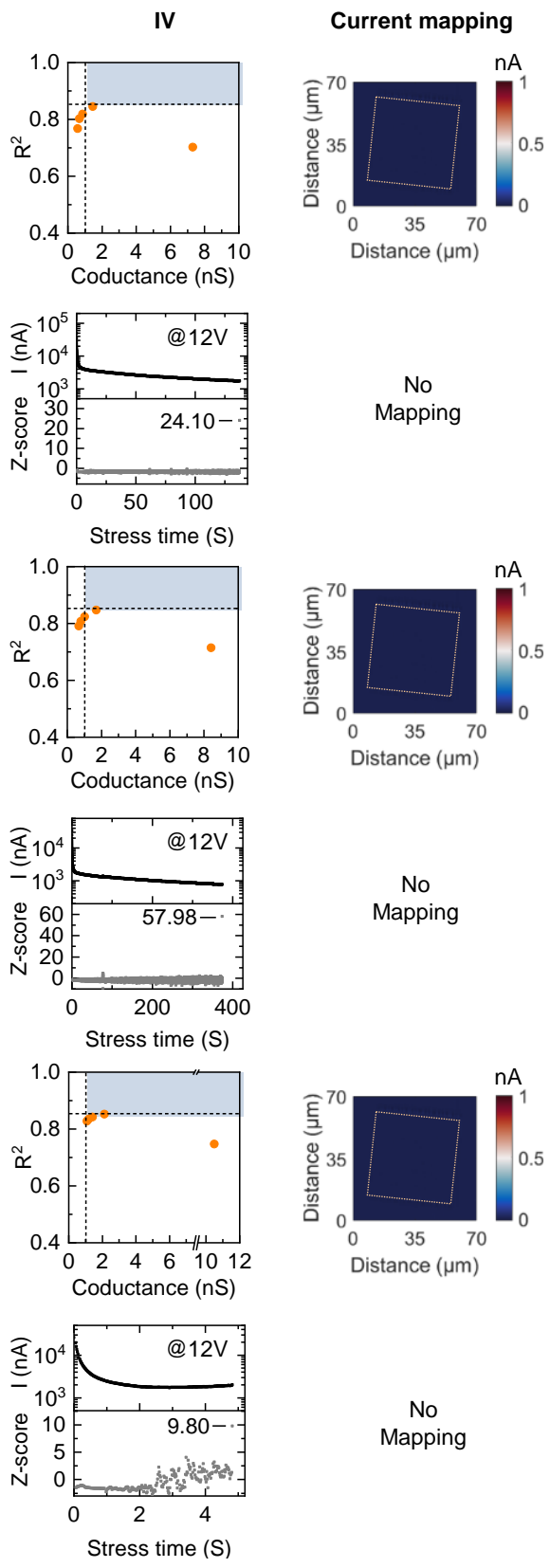

Microscope

$$
\begin{gathered}
\text { No } \\
\text { Mapping }
\end{gathered}
$$
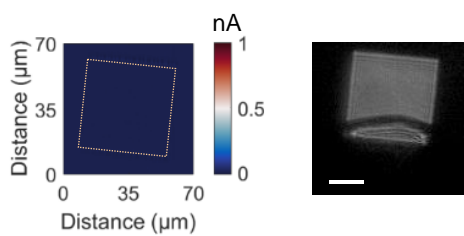

No Mapping
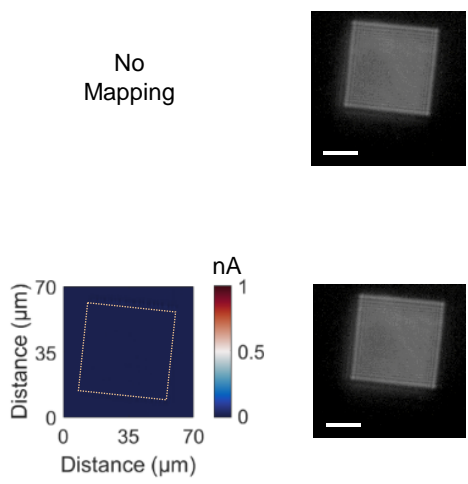

No

Mapping
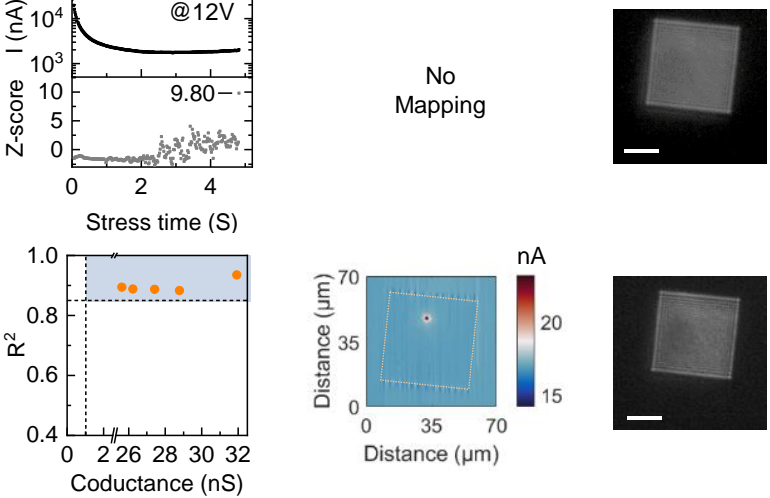

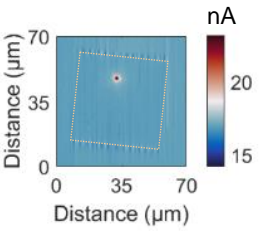

Figure S2. Another sample's fabrication process. The first column is the electrical monitoring of nanopore formation (Shaded area of the scattering plot is the true positive initial breakdown); the second column is the laser enhanced ionic current mapping of the $\mathrm{SiN}_{\mathrm{x}}$ membrane (The laser power is $6 \mathrm{~mW}$, and the biasing voltage is $200 \mathrm{mV}$, mapping step size is $500 \mathrm{~nm}$ ); the third column is the microscope image of the $\mathrm{SiN}_{\mathrm{x}}$ membrane; 
Supporting Information

Sample \#1

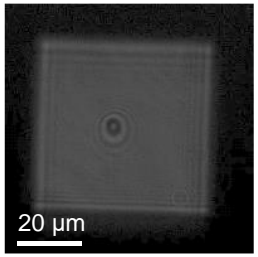

\#6

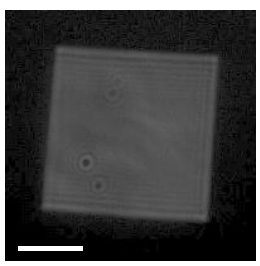

\#11

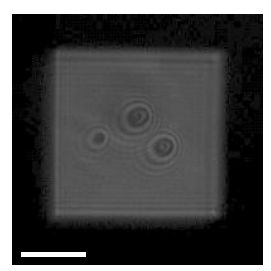

\#2

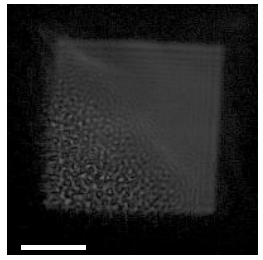

\#7

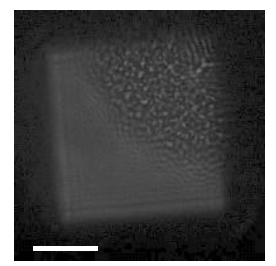

\#12

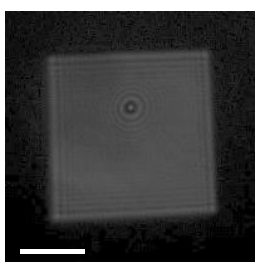

\#3

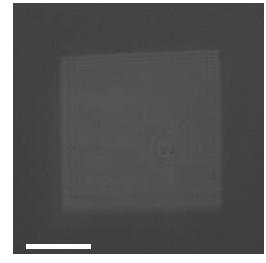

\#8

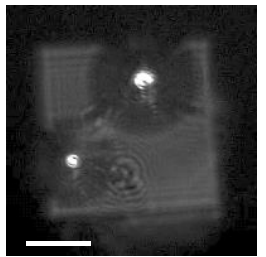

\#13

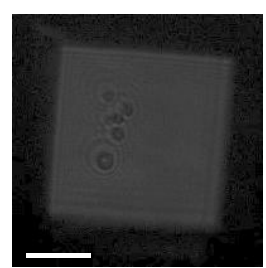

\#4

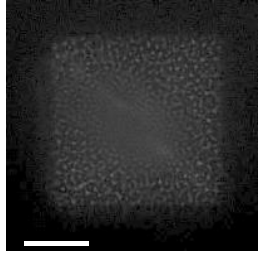

\#9

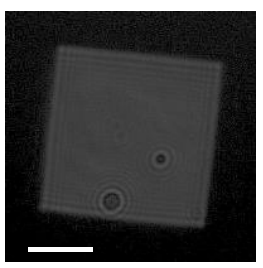

\#14

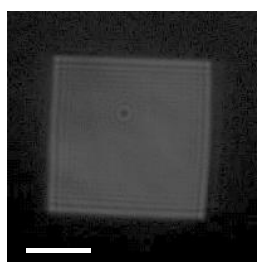

\#5

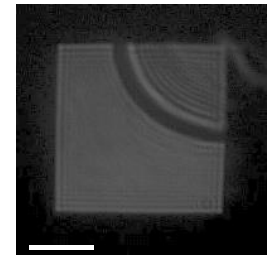

\#10

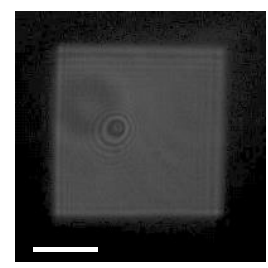

\#15

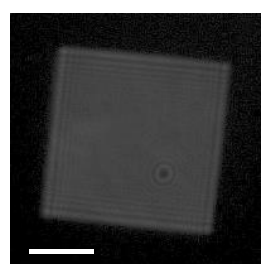

Figure S3. Microscope images of the redox induced bubbles from 15 samples. The bubbles show random distribution and morphology. 


\section{Supporting Video S1.}

Redox induced bubble generation. The real-time paced video is composed of 8 representative samples (Sample \#1, \#4, \#6, \#7, \#11, \#12, \#13 and \#14). Both single bubble and multiple bubbles were observed. 